\title{
Collaboration, Consultation, or Transaction: Modes of Team Research in Humanities Scholarship and Strategies for Library Engagement
}

\section{Megan Senseney, Eleanor Dickson Koehl, and Leanne Nay}

\begin{abstract}
With the rise of digital scholarship, humanists are participating in increasingly complex research teams and partnerships, and academic libraries are developing innovative service models to meet their needs. This paper explores modes of coworking in humanities research by synthesizing responses from two qualitative studies of research practices in the humanities and proposes a taxonomy of multiperson research that includes collaborative, consultative, and transactional research partnerships among scholars, graduate students, academic staff, and a range of other potential stakeholders. Based on an analysis of humanities scholars' self-described research behaviors, we provide recommendations for academic librarians who are developing and sustaining service models for digital scholarship.
\end{abstract}

\section{Introduction}

During the last decade, libraries have invested significantly in service models for digital scholarship, particularly digital humanities (DH). Models for library-engaged digital humanities often emphasize research collaborations where the participating librarian becomes a member of a research team. This focus mirrors the way in which collaboration is described as a defining characteristic of digital humanities, due in part to the fact that many DH projects require human and material resources spanning disciplinary and institutional boundaries. Collaborative digital humanities projects have also been encouraged through the proliferation of grant programs that incentivize collaboration, such as the multinational Digging into Data initiative, the National Endowment for the Humanities' collaborative research grants, and the recent Humanities Without Walls (HWW) grand challenge grants funded by the Andrew W. Mellon Foundation.

The Taxonomy of Digital Research Activities in the Humanities (TaDiRaH) offers a broad definition of collaboration, which is "any research activity being done jointly by several researchers, possibly in different places and at different times." ${ }^{1}$ Yet there are many models within

\footnotetext{
${ }^{*}$ Megan Senseney is Department Head in the Office of Digital Innovation and Stewardship at University of Arizona Libraries; email:msenseney@email.arizona.edu. Eleanor Dickson Koehl is Digital Scholarship Librarian at HathiTrust; email:efdkoehl@hathitrust.org. Leanne Nay is a Digital Engagement Librarian in the Scholars' Commons of Herman B. Wells Library at Indiana University Bloomington; email: Inay@indiana.edu. @2019 Megan Senseney, Eleanor Dickson Koehl, and Leanne Nay, Attribution-NonCommercial (http://creativecommons.org/licenses/by-nc/4.0/) CC BY-NC.
} 
academia for scholars and academic staff coming together around a research question, from scientific labs to research support provided by archivists and special collections librarians. Overly broad use of the term "collaboration" within academic libraries masks the variety of research behaviors and needs that exist for partner-based research support and undermines the library's ability to anticipate users' needs, develop rightsize service models, and effectively steward limited resources. Nevertheless, the nuance of when and how humanities scholars work in tandem with others remains underexplored within the profession.

This lack of understanding poses a challenge for libraries and academic research centers, including digital humanities centers, as they develop models for engaging in research activities that span a continuum of time and resource investment. Vinopal and McCormick have emphasized that not all library-engaged digital scholarship has to involve the same level of support, leading New York University's libraries to implement a tiered service model. ${ }^{2}$ While NYU's model primarily focuses on the technical complexity of a project, better understanding the human complexity (that is to say, the ways in which humanities scholars involve others in their research) would further help libraries develop the kinds of services and infrastructure that researchers in the humanities most need. Likewise, existing programs that aim to incentivize collaboration could be modified to accommodate a deeper understanding of the nature of group-based humanities research, manage expectations, and improve evaluation. Overall, clarifying models for research partnerships in the humanities is a necessary starting point for uncovering underlying issues and developing more effective institutional policies in support of the collaborative enterprise, as well as providing scaffolding for more productive project processes and outcomes.

This paper seeks to understand how libraries engaged in digital scholarship might effectively adjust their service models to support the full range of research interactions that scholars describe as necessary to their work. To better articulate the division of labor in humanities research, we synthesize responses from two studies of research behavior and explore when and how scholars in the humanities and humanistic social sciences work with others. In the HWW study, the collaborations were primarily among faculty at institutions of higher education, with graduate students often included on project teams. Collaborations involving librarians along with faculty and graduate students were more strongly represented in the User Requirements Study (URS), though neither study was explicitly designed to elicit examples of librarian-faculty collaboration.

We characterize research practices using in vivo depictions derived from interviews to highlight the motivations and challenges of DH scholarship, and we propose a taxonomy of multiperson humanities research that includes collaborative, consultative, and transactional research partnerships. This taxonomy describes both how the actors within the partnership relate to one another and how they exchange information. By developing a finer-grained taxonomy of multiperson humanities research, this paper will accomplish the following aims: (1) contribute to library and academic units' understanding of collaboration and support for humanities research; (2) help project partners develop functional project charters and work plans; and (3) assist funding agencies and other administrators in assessing the viability of proposals and project outcomes for collaborative work in the humanities.

\section{Literature Review}

In a 2006 report on cyberinfrastructure, the American Council of Learned Societies highlighted collaborative research within digital scholarship as a motivating requirement for ongoing de- 
velopment of shared technical infrastructures, ${ }^{3}$ and recent literature continues to explore the vital relationship between collaboration and infrastructure. ${ }^{4}$ Other studies of digital humanities have focused on social aspects of collaboration such as team membership, ${ }^{5}$ the importance of face-to-face interaction for distributed teams, ${ }^{6}$ and credit and coauthorship in cross-boundary collaborations. ${ }^{7}$ In 2012, an entire book of essays on the topic of collaborative research in DH spanned a range of issues from collaborative crowdsourcing to standards making. ${ }^{8}$ While most publications on DH collaboration have been postproject assessments by team members, there have also been some empirical studies that have included discussions of institutional interventions in the collaborative process, with an article on the Digging into Data initiative, ${ }^{9}$ a series of articles annually documenting the collaborative practices of the INKE project, ${ }^{10}$ and a postmortem on Project Bamboo ${ }^{11}$ serving as notable exemplars.

As interest in digital humanities (and digital scholarship more generally) has gained momentum, academic libraries have made concerted efforts to establish their role within the evolving research landscape. ${ }^{12}$ Focusing on questions of scale and sustainability, librarians at New York University proposed a tiered service model for supporting digital scholarship in research libraries. ${ }^{13} \mathrm{~A}$ complementary approach that shifts emphasis away from service models is one of greater library-faculty collaboration and partnerships, ${ }^{14}$ as well as more intensive engagement through embedment in academic departments. ${ }^{15}$ At the Maryland Institute for Technology in the Humanities and the Scholars' Lab at the University of Virginia, emphasis has shifted still further away from service toward librarian-led research and development on digital humanities projects. ${ }^{16}$ To enhance librarians' ability to engage in intensive DH research and collaboration, initiatives such as Developing Librarians have created opportunities for librarians to learn new skills and competencies. ${ }^{17}$ The number of different approaches to the question of how libraries should engage in digital humanities reflects the spectrum of current attitudes toward the role of librarians in the research process and how best to meet the mission of the academic library.

While digital humanities is a particularly useful space for interrogating questions of collaboration and other modes of multiperson research, it also maps to a broader trend in the humanities and the academy at large. Humanities Without Walls and the Central New York Humanities Corridor are two examples of recent initiatives to foster collaborations among the institutional partners of a regional consortium, ${ }^{18}$ and the National Endowment for the Humanities has developed programs dedicated to funding collaborative research. ${ }^{19}$ Reflections on the importance of collaboration surface in discussions of the New Humanities, ${ }^{20}$ public humanities, ${ }^{21}$ and the sustainability of humanities research. ${ }^{22}$ Across these discussions, a strong emphasis on interdisciplinarity and cross-organizational engagement emerge as central themes, which connect the collaborative humanities into the wider literature on organizational management, communication, and the science of team science..$^{23}$ The trend toward collaboration in the humanities is consistent with a broader administrative interest in collaborative interdisciplinary research throughout higher education. ${ }^{24}$ Its perceived benefits include the likelihood of producing more innovative, impactful scholarship and a more equitable distribution of the burden of knowledge across research teams. ${ }^{25}$

\section{Methods}

This paper brings together findings on collaboration from two qualitative studies of research practices in the humanities. The first study (HWW) was conducted from March 2015 through 
October 2016 and comprised a set of 25 semistructured interviews with recipients of the first round of Humanities Without Walls grand challenge grants. The 14 funded projects spanned a wide range of topics in the humanities and humanistic social sciences intended to touch upon the broad theme of the "Global Midwest." Project proposals were required to include collaborative research teams composed of partners from two or more institutions within the HWW consortium as a condition for funding. The digital complexity of awarded projects varied considerably, but all funded projects included digital components for research and communication. Using a census sampling method, the team sought interviews with all 82 scholars participating in one or more funded projects, resulting in a 30 percent response rate. The interview protocol was iteratively developed by a team of four investigators by developing a set of 11 questions that mapped to two or more explicit project goals. Each interview lasted approximately one hour, and questions focused on respondents' research interests, their characterizations of collaborative structures in HWW, their strategies for data sharing and scholarly communication, and their thoughts on potential impacts and future outcomes (see appendix A). The team recorded, transcribed, and coded the interviews in ATLAS.ti 7. Preliminary codes were developed inductively based on themes identified in the transcripts, and each transcription was coded twice to ensure intercoder reliability. This study applies a method that draws upon a theoretical grounding in qualitative content analysis. ${ }^{26}$

The second study, the User Requirements Study (URS), was designed to understand the needs of scholars and librarians interested in conducting computational text analysis with the collection of digitized text amassed by the HathiTrust consortium. From April 2015 to November 2016, researchers conducted 18 semistructured interviews with information professionals and researchers from various humanities and humanistic social science disciplines whose work incorporated text analysis and, for some, also used HathiTrust resources. Interviews covered scholars' research practices; strategies for obtaining and analyzing data; publication methods; and resource needs (see appendix B). Using a purposive sampling method, participants were recruited from attendees at professional conferences (including the HathiTrust Research Center UnCamp, the DLF Forum, and the Chicago Colloquium for Digital Humanities and Computer Science) and from active members in HathiTrust Research Center User Group forums and activities. Qualitative analysis proceeded using a similar content analysis strategy as the HWW study, including inductive codebook development followed by multiple rounds of coding to ensure intercoder reliability.

The studies were conducted independently by two different research teams, but membership overlapped significantly. The authors of this paper represent a subset of the two research teams. Following the initial studies, they subsequently combined the interview data from both and conducted another round of qualitative content analysis based on two sensitizing concepts: motivations for group-based research and the types of interactions between group members. The combined data represent 43 total interviews following two different protocols designed for two different purposes, but both touch on features of group work in humanities contexts in interesting ways. The findings and discussions that follow highlight important features of multiperson work that were present across both studies. In constructing typologies for motivations and relational structures, the team sought outliers and negative cases that contradict the emerging model, and none were found; however, findings may be limited by the nature of the studies. In both cases, interviews are reliant on self-reporting and subject to the effects of social desirability. Recruiting followed sampling strategies that met the purposes of 
the original studies and may not be representative of humanities scholarship more generally. Despite its limitations, a combined analysis of two studies focused on innovative research in the humanities provides useful insights into key professional aspects of how such research efforts are organized and negotiated in multimember contexts.

\section{In vivo Characterizations of Multiperson Humanities Research}

Interview questions from both studies addressed the theme of collaborative scholarship and, in both cases, elicited responses that characterize the nature, duration, and motivating factors for study participants to engage others in their research. Additionally, interviewees described the challenges and disincentives for collaborative or multiperson scholarship. Despite the diversity of projects and research methods described in the interviews, responses gathered during the two studies shed light on several key attributes of group-based humanities research. These attributes can be divided into four themes: addressing skills gaps, capacity building, scholarly communication, and project logistics. In this section, respondents' own in vivo language characterizes their work to illustrate these attributes.

\section{Skills Gaps}

\section{"To leverage each other's strengths"}

A primary motivation for participating in multiperson humanities research is to fill skills gaps, specifically in areas such as computer programming, statistics, or project management. For some, these partnerships take the shape of project teams where each member is able to contribute individual expertise. For example, one respondent said, "In my ideal project, I would have a team of researchers, a couple of them are experts on [one area of the project], and then I would also bring in another person who understands [another area of the project]. So I can see a team of three or four scholars working together to leverage each other's strengths" (URS P2). The theme of working with other researchers with complementary expertise was echoed in 21 of 43 total interviews.

\section{"I'll just hire someone to do it"}

The HWW interviews reveal that, for many, the purpose of seeking grant funding is to have the means to hire people with a particular skill set that is different from or more advanced than their own. This expertise can make the project technically feasible or help meet deadlines, but the focus on hiring also shifts attitudes from "leveraging strengths" to delegating responsibilities. As respondent HWW P16 explained, "I don't think it's a good use of my time in collaboration worrying about the actual programming when there are people more qualified to do that than myself." Such for-hire research support was also present in URS responses, with one participant saying with regard to programming tasks, "If there's something that I feel like is a well-defined task I'll try to get someone to do it and I'll just hire someone to do it" (URS P16).

In addition to support for computational research, respondents in both studies expressed a need for assistance with activities they could delegate such as scheduling, sending emails, or disseminating information among their project team. This type of administrative work was described as mundane and time-consuming. One respondent explained that many researchers do not have time off to participate in these types of projects, and organizational tasks are potentially more time-intensive than the research itself (URS P15). Hiring a project coordina- 
tor was considered prohibitively expensive for some, but advantageous if possible. As one respondent said, "I think that the single best decision that I made... was bringing on board a really great project manager" (HWW P5).

Stratifications based on hiring can also shape whether or not individuals within a given team perceive themselves as members of a collaboration. One URS interviewee pushed back against the suggestion that his research team had collaborated on a project, saying, "So, yes, that's a very intense collaboration, but you wouldn't call it collaboration when you're actually working, when you're paid to do these things" (URS P15). In another case, certain partners in a project were not considered collaborators but were "meant to actualize someone else's vision. They were hired" (HWW P14). The tension over what is and is not a collaboration was also reflected in the way some respondents found it difficult to discuss their projects as collaborations. As one interviewee said, "I can tell you based on [...] two years coordinating this, I have no idea what collaboration looks like. I have no idea if our collaborators have any idea what collaboration looks like" (HWW P29).

\section{"Let's work with you and teach you how to do it"}

While some researchers seek partnerships with others to complement their own skills and others hire with an eye toward delegation, a third strategy emerges in which researchers build partnerships when they want to learn the skill themselves. Some librarians or other support staff who partner on research projects perceive their role as helping scholars develop functional skills to carry out tasks on their own. One URS respondent said of library-based text analysis consultations, "optimally we would like to be able to say, 'this is what we recommend doing, let's work with you and teach you how to do it'" (URS P10). Among respondents, librarians seemed most attuned to partnership via training (URS P13 \& P12). Such skill building among partners can also be a secondary outcome of research collaborations. One participant in the HWW interviews described the way a "big collaboration" with another expert had led to her becoming proficient in a new skill set. She said, "I would just take my graduate assistants, and I would just fly out to [location redacted] and sit in the lab for long weekends and work on the project, which is how I learned about digital design, having to do it" (HWW P16).

\section{Capacity Building}

"We had an existing network already in place that we wanted to build upon"

A second major theme focused less on individual skills and more on developing expertise and community around a given topic. A majority of HWW respondents described sustaining or creating a network, sometimes through in-person interactions, as their primary motivation for participating in their collaboration, and interview participants described the way they used the HWW funding opportunity to build on those pre-existing relationships. As one interviewee said, "We had an existing network already in place that we wanted to build upon to do a particular research project. And we also hoped that ultimately the project itself would help strengthen our network and there would be a lasting benefit" (HWW P6). Two respondents described professional collaborations that developed from personal relationships, where the existing relationship was seen as advantageous to their collaboration (HWW P3 \& P4). Participant HWW P24 said, "So the personal relationships were there before we started, and then one thing I think that was quite important was that we met twice before the formal activities began." 
"What's really nice on our campus is they are building up the expertise" Interviewees reported variant understandings of the library or campus digital scholarship center as an expert resource for DH research projects. In the URS, librarian participants in particular described the way they have provided research support for text-mining projects. Their responses showed that research centers have tried to build capacity for projects by cultivating expert staff, with one head of a digital scholarship center saying, "For a long while, whenever we did this kind of work we would usually go out and find ourselves a computer scientist or a mathematician... and get them to work with us on projects. Now what's really nice on our campus is they are building up the expertise, the people like [name redacted] who we can go to help with work that we do" (URS P4). Still, some advanced researchers do not see their local librarians as having adequate expertise to partner with them on their research. One text analysis scholar said, "I think [my university] recently built a center for digital scholarship, but it really needs a [Natural Language Processing] expert if it wants to be of assistance to those who are trying to do serious work. I mean, experts to whom grad students in [computer science] would go for advice" (URS P6). This researcher instead turned to other scholars or local experts for assistance. In general, researcher interviewees in both studies made scant mention of librarians or library support.

\section{Scholarly Communication}

"You have to develop a common language"

Communication, transparency, and credit also emerged as common challenges that shaped how team members perceived one another. The research partnerships described in both studies were often interdisciplinary, and learning to navigate disciplinary divides was a common theme expressed in both sets of interviews. One participant, after describing the various backgrounds of his collaborators, said, "Already you can see we come from rather different backgrounds and there are ways in which you have to develop a common language" (HWW P24). Working across domains was seen as a beneficial characteristic of collaborations. One participant said, "Collaborations give us this kind of rich multiplicity of views and understanding" (HWW P26).

For some, the library was seen as a space that spans campus boundaries where interdisciplinary relationships can be brokered. One respondent described the library as "the one functioning interdisciplinary space on a university campus" (URS P8). Speaking as a librarian, another participant explained, "we try to get that deal brokered, and a lot of times I'll do things like connect a scholar with somebody over in [computer science] and just make sure that the communication's really happening because sometimes there's a little cultural divide that separates humanists from computational folks" (URS P12).

Nevertheless, research teams are not always interdisciplinary. URS participants were at times skeptical of working with computer scientists or statisticians, and some text analysis research groups prefer to develop the requisite skills to carry out their projects in researchers with subject-matter expertise as opposed to drawing on collaborators across departments or fields of study. As one respondent explained, "I find it much easier to bring a humanist along and teaching them enough computer science to be dangerous than trying to get a computer scientist to understand the humanities" (URS P15).

"Humanities scholars have a tradition of holding their ideas quite close to their vests" Multiple respondents remarked upon the perceived individual nature of traditional humani- 
ties work. They were unlikely to self-identify as a "lone scholar," but several portrayed their colleagues as isolationists. One respondent described himself as being unique among humanists because they had participated in collaborative projects before, and another respondent observed that "humanities scholars have a tradition of holding their ideas quite close to their vests" (URS P12). In general, humanities scholars were noted as being less familiar with collaboration than other disciplines. One respondent with a background in linguistics reported that linguists are much more accustomed to collaboration than other humanities scholars because "you really can't do good linguistics of this sort unless you're working with other people" (HWW P7).

A culture of sharing, or lack thereof, was another key problem in humanities research that was raised by one respondent: "But I think that's another major problem in the humanities we run into all the time where, in some of the sciences, not all, there's kind of a culture of sharing. I want to share my data. I want to share my dataset. I have to share my dataset so you can do reproducibility. There's a real sense in the humanities where, no, this is my data nobody's going to touch it" (URS P4).

\section{"Collaboration was built around the idea that both parties would be equally ac- knowledged"}

As respondents discussed collaborative initiatives, many were mindful of the importance of providing appropriate credit and recognition for project partners. One respondent noted, "for us, the notion of collaboration was built around the idea that both parties would be equally acknowledged" (HWW P21). Another discussed acknowledgment for contributors who are not formally designated project partners, "we personally think that it's important that those who contribute to digital projects are acknowledged as contributors---as helping with the digital project as behind the screen help, but in fact contributors in the truest sense" (HWW P21). Negotiating appropriate credit, however, also revealed moments of tension within the projects. One respondent mentioned, "there was a little bit of misunderstanding and some disagreements ... had to do with who is being acknowledged for what" (HWW P6). Interviewees also discussed the boundaries surrounding student collaborators. One participant remarked that the students involved in his project were no longer "just graduate students" but could be considered, "fellow collaborators in the project at this point" (HWW P24). Such a statement presumes, at least for this individual, the value of crediting student labor as collaborative as a way of acknowledging their effort. Respondents among the HWW awardees in particular were quick to discuss concerns regarding how graduate students were employed and engaged within a broader project, suggesting unresolved tensions regarding students' roles in multiperson research.

\section{Project Logistics}

"Collaboration works best when each of us kind of has something to get out of it" Respondents in both studies talked about the give-and-take of research partnerships. Some felt that collaborations are most successful when each member has equal stake in the project. One interviewee reflected, "As I see it, particularly in the digital realm, it seems to me that collaboration works best when each of us kind of has something to get out of it-specific to get out of it and specific to put into it" (HWW P25).

Multiple respondents identified a difference in priority among their collaborators as a significant barrier. This inconsistency affected how team members communicated, scheduled 
in-person meetings, and shared responsibilities. According to one respondent, "In order for two people to collaborate the priority has to be the same for the project and the availability of time to do it has to be the same" and "when everybody's busy... sometimes the only way to get people's attention is to put money on the table" (URS P2).

As a whole, the language used by respondents illustrates this varying level of commitment among project teams. Some respondents from the HWW study spoke confidently about their roles and involvement in their projects, while others were quick to point out that they were not key personnel and did not have a clear motivation for participation. The URS interviews also showed discrepancies in how respondents spoke about their contributions to various projects. In general, respondents were reluctant to label themselves as collaborators when discussing anything beyond their primary research. The use of mine, ours, or theirs throughout these interviews when discussing research projects suggests the varying levels of commitment.

\section{"There are a constant set of bottlenecks in the collaboration that tend to arise"}

Respondents also indicated that retaining team members throughout their projects was a notable challenge, especially when working with graduate students whose work is term-limited. When describing this cycle, one respondent said, "those of us in the humanities kind of have to reinvent the wheel in terms of finding somebody new" (HWW P16). In reflecting on this problem, the respondent focused on the "bottle neck" of graduate student support. They explained that, without a solid departmental infrastructure to support graduate students, it could be challenging to find a replacement after students graduated. The respondent recommended that "we need to be more like the sciences, not less like the sciences, where there are allowances made for graduate students and then the graduate students are treated as integral collaborators in a research project and not just doing busy work" (HWW P16). For one respondent, the limited number of hours that graduate students were allowed to work also made it difficult to complete the project (HWW P3).

\section{"Collaborations between institutions: much more difficult"}

Once respondents assembled their project teams, working across institutions and navigating differences in infrastructure was another hurdle. While HWW projects were required to be interinstitutional, the challenge of working with colleagues at other institutions was identified in both sets of interviews. One respondent was overwhelmed by the operational differences in disseminating funds across three institutions (HWW P15). Another referenced the variation in institutional review board procedures (HWW P12). According to one respondent, "Normally in the humanities, we're accustomed to internal grants that fund our own time and our own expenditures, but having to work cross-institutionally has been a challenge. It put back our progress" (HWW P3). A URS interviewee said, "Collaborations between institutions: much more difficult. And...there's money, there's institutional blockages, and then anything over half a dozen people, it gets complicated very quickly. And so the people dynamics get very complicated" (URS P15).

\section{"Collaboration means collaboration over time"}

Finally, finding time to participate in "extra" commitments was a repeated concern raised by respondents. As one faculty member explained, "We don't have time off to do this... I still have all my committee work to do. I still have my teaching to do. I still have all my responsibilities 
there and this is all extra time and the organizational work is pretty daunting" (HWW P15). A majority of HWW respondents were disappointed with short timelines, especially when working with new collaborators. Another respondent explained, "collaboration means collaboration over time. You know, you have to be able to develop your relationship over time and you can't from one day to the next throw people together" (HWW P24). Another highlighted the fact that timelines can change throughout the course of a project, and original timelines often prove to be unrealistic (HWW P25).

Moreover, respondents described the way research partnerships can be fleeting or flexible over time. One HWW interviewee said that collaborations can vary between a "50/50 shared enterprise" to other ratios, and also may be "collaborative at one point in the project and not at others" (HWW P5). Such a depiction highlights the way that collaborations are nonstatic relationships.

\begin{tabular}{|c|c|}
\hline & $\begin{array}{c}\text { TABLE } 1 \\
\text { Taxonomy of Multiperson Humanities Research }\end{array}$ \\
\hline Collaborative Rese & earch Mode \\
\hline Description & $\begin{array}{l}\text { In which a group of researchers work together with shared decision making and } \\
\text { multidirectional information exchange. This form of research intends to draw on the } \\
\text { strengths of all parties in the collaboration. }\end{array}$ \\
\hline Key Characteristic & Multidirectional information exchange \\
\hline Example & $\begin{array}{l}\text { A group of four researchers from different institutions develop a grant proposal to } \\
\text { build on existing research interests and activate a network of scholars around a central } \\
\text { idea. The researchers pursue a project wherein all partners contribute their expertise } \\
\text { and time to reach a common goal. The partners are equally involved in key decision } \\
\text { making and they receive recognition commensurate with their contributions. }\end{array}$ \\
\hline Consultative Resea & arch Mode \\
\hline Description & $\begin{array}{l}\text { In which researchers seek assistance from an expert who advises on a limited basis } \\
\text { and with unidirectional information exchange. Often the researchers recognize } \\
\text { they lack expertise in an area of their project, and generally the consultation serves } \\
\text { to develop their skills such that they can work with less assistance in the future. }\end{array}$ \\
\hline Key Characteristic & Unidirectional information exchange \\
\hline Example & $\begin{array}{l}\text { A researcher uses the support services of her local digital scholarship center to improve } \\
\text { preliminary code she wrote for a text analysis project. A research programmer in the } \\
\text { digital scholarship center edits and repackages the scholar's code and then, via pair } \\
\text { programming, assists the scholar in implementing the revised code in her project. The } \\
\text { research programmer hopes that this process teaches the researcher skills in object- } \\
\text { oriented programming so that she can work independently in the future. }\end{array}$ \\
\hline Transactional Re & earch Mode \\
\hline Description & $\begin{array}{l}\text { In which researchers offer payment, frequently monetary, to have a component } \\
\text { of their research done for them over the course of a defined time frame and } \\
\text { where services, as opposed to information, are exchanged. This form of multiparty } \\
\text { research can be considered work for hire. }\end{array}$ \\
\hline Key Characteristic & Services exchanged \\
\hline Example & $\begin{array}{l}\text { A scholar who engages in data-driven research occasionally relies on the services of } \\
\text { others to carry out her analysis. When she feels low on time, she will hire someone } \\
\text { to complete a well-defined task that helps her advance her research project. }\end{array}$ \\
\hline
\end{tabular}




\section{Taxonomy of Multiperson Humanities Research}

Based on findings from our combined analysis, the taxonomy presented in table 1 describes the modes in which researchers work in partnership with one another on digital humanities research projects. Multiperson digital humanities research generally takes one of three main forms: collaborative, consultative, or transactional. Participants in a research project may move among these modes through the duration of the project's life cycle. The examples for each mode are drawn directly from the two studies.

\section{Discussion}

As libraries continue to investigate different modes of user engagement and evaluate existing service models, it is important to attend to the motivations cited by scholars in the humanities for seeking research partners and the particular challenges they face. Notably, the vast majority of nonlibrarian respondents in both the User Requirements Study and Humanities Without Walls Study did not mention any type of interaction with the library, which may indicate that library participation is being overlooked or is altogether absent from these projects. It may also suggest that the services provided by librarians are not valued as "serious work" or that scholars are not sufficiently aware of the broad and deep expertise located in the university library. And while the librarians interviewed for these studies tended to prefer to be involved in DH projects either in the collaborative or consultative modes, scholars in the studies often stressed the value of turning to others to contribute to their research as a transactional service. This discrepancy suggests the importance of clarifying, implementing, and widely communicating the actual and potential roles libraries play across all three modes of research: collaborative, consultative, and transactional. To ensure that libraries are situated as part of the solution to the challenges of group-based humanities research, we provide the following recommendations:

1. Know your environment. Periodic needs assessment is a necessary component of digital scholarship services, both for developing new library models and evaluating established ones. In the process of assessment, librarians will want to learn about current and potential projects across the institution with an eye toward perceived skills gaps. Librarians are well positioned to serve as knowledge brokers across disciplinary boundaries, either helping to establish new connections among researchers or developing locally relevant training modules to help researchers learn methods, approaches, and technologies adopted from other fields.

2. Know your limits. Internal workforce assessment and strategic planning is equally important. Librarians face a number of resource constraints that limit their service models and force pragmatic choices. Following Vinopal and McCormick's tiered services model, it is reasonable to establish baseline services and to articulate conditions for enhanced support and applied research and development. ${ }^{27}$ These may include strategic library investments, joint applications for external funding, or direct payment for services rendered. Standard services may vary according to the mission of the library, the subject and functional expertise of the library staff, and constraints on time and financial resources.

3. Leverage your institutional expertise. Navigating the institutional components of larger projects has proven particularly challenging for the scholars we interviewed, with issues ranging from establishing research infrastructures to navigating the logistics of 
sponsored projects. Providing guidance on project management and administration or helping to design and implement project work plans is a useful service that also creates opportunities for early interventions in downstream issues related to data management and scholarly communication.

4. Model best practices. Without engaging directly with any given team, librarians can also provide valuable support by addressing the work of multiperson humanities scholarship as an information problem that requires access to information resources. These may take the form of model project charters and sample MOUs; guidelines for credit and acknowledgement; and recommendations for managing research materials in the context of computer-supported cooperative work..$^{28}$

The results of these two studies demonstrate the range of partnership models that the library may seek to accommodate. Rather than advocating for one form over another, we encourage strategies that embrace this variety and articulate the circumstance under which librarians participate in each. As one respondent was quick to point out, not all libraries need to build digital humanities centers (URS P8), but there are still ample opportunities for librarians to engage in $\mathrm{DH}$ partnerships. The following section makes recommendations for librarians to be involved in $\mathrm{DH}$ partnerships, broken down by the modes described in the taxonomy for multiperson research.

\section{Collaborative Research Mode}

In the collaborative mode, subject and functional librarians may be able to apply their expertise and engage in joint decision making with fellow scholars. This type of partnership may be especially relevant at institutions where librarians are academic faculty and must meet tenure requirements. These relationships may extend over long durations with varying levels of effort over time. Regardless of whether a given project is formally funded, these kinds of relationships are best articulated through a project charter or a memorandum of understanding.

\section{Consultative Research Mode}

In this mode, librarians act as intermediaries and help to make connections between the researcher and resources. They meet with researchers, discuss their projects, and make recommendations for tools, resources, and services to advance their projects. For example, librarians may provide drop-in consultation hours or instructional sessions with a focus on topics such as text analysis, data visualization, or copyright. Consultative engagement is a natural extension of traditional reference and user service models and is often situated as the foundation of a tiered services model for digital scholarship. It also remains the most preferred mode of engagement among the library-based participants in this study.

\section{Transactional Research Mode}

Libraries could also reserve a cache of hours for one-off projects, explore fee-based services, or help facilitate a transactional partnership. For example, librarians may be tapped for their particular expertise to develop a custom metadata schema; deal with data processing issues; or other one-time contributions to a research project. Alternatively, a librarian may coordinate the digitization of collections or manage a group of students hired to complete specific tasks. Outside library publishing and reproduction services, transactional arrangements for digital scholarship may represent new territory, and further research is warranted to develop ethical 
and sustainable policies and protocols for integrating transactional labor within a broader service model.

\section{Conclusion}

Better understanding of humanities scholars' emerging research practices is crucial for strategic planning and outreach around digital scholarship. Despite increased interest in collaboration within digital humanities and across institutions of higher education, collaborative research remains underspecified and is often conflated with other forms of group work. By adopting a formal taxonomy for modes of multiperson research, librarians are better able to articulate the types of services they provide and the resources required for different modes of engagement with scholars. Transparent communication regarding transactions, consultations, and collaborations within the library can assist with managing expectations and diffusing tensions when negotiating librarian-faculty partnerships.

\section{Acknowledgements}

The authors would like to gratefully acknowledge Harriett Green, Angela Courtney, Maria Bonn, Nicholae Cline, Sayan Bhattacharyya, Robert McDonald, Justin Williams, Krystal Cooper, and Alex Kinnaman for their collaborative efforts on the initial HWW and URS studies; the Humanities Without Walls Consortium and the HathiTrust Research Center for supporting the research; Alix Keener and Courtney McDonald for providing thoughtful feedback on early drafts; and the interview participants who made this research possible. 


\section{APPENDIX A. Interview Guide for Humanities Collaborations and Research Practices: Exploring Scholarship in the Global Midwest}

Estimated length: 45 to 60 minutes

\section{Goals of the Interview}

- Identify how scholars participate in research practices and how collaboration affects their research processes

- Determine how humanities scholars define collaboration and the types of informationsharing workflows and research infrastructures they build in collaborative research

- Understand the impact of humanities initiatives focusing on collaboration, like the Global Midwest, and how they expand the global impact of scholarship in the humanities

\section{Interview Questions}

\section{Background}

1. What is your position and area of research?

2. Please briefly describe the focus and goals of your HWW-funded Global Midwest project.

3. Structures of Collaboration

4. How did you initially envision the "collaboration" element for your project? How do you define "collaboration"?

5. What tools did you decide to use for managing project workflow processes between your collaborators? What would help make the process smoother/what do you need?

6. What are the formats/methods you are considering for publishing your project results?

7. Data Sharing and Scholarly Communication

8. How are you sharing/disseminating updates about your ongoing project?

9. How has this collaborative project affected the ways that you share research, store data, or conduct your research in general?

10. Where and how are you storing the data for your project?

11. What kinds of challenges did you encounter during this project?

12. Scholarly Impact and Future Outcomes

13. How do you envision the impact of your project work and publications?

14. Do new platforms and tools open up possibilities for changed scholarly publications and research outputs in the humanities? Could you elaborate? 


\section{APPENDIX B. Interview Guide for User Requirements for Textual Analytics}

Estimated length: 40 minutes

\section{Goals of the Interview}

- To ensure that the services that the HathiTrust Research Center (HTRC) develops presently and in the future are adequate to the needs of the users

- Develop a suite of general services for HTRC users through the Scholarly Commons

- To obtain ideas for illustrative use cases for use in workshops and tutorials

\section{Interview Questions}

\section{General Information}

1. Describe your research area and interest in text analytics.

Prompts

- How did you first become aware of the potential for text analytics in your research?

- At which stage(s) of your research do you employ textual analytics?

2. Describe the relationship between your research question and the text analytic methods and approaches that you use.

Prompts

- How do you determine what methods and approaches to use for your research?

- How does textual analytics fit into your methods?

- At what point in your research do you use analytics? (for example, to frame research questions, for exploratory work, for confirmation/disconfirmation of your interpretive work?)

- When might you use text analysis in conjunction with other methods and approaches in your discipline?

3. Where/to whom do you go for assistance when applying the textual analysis algorithms? Prompts

- Could you tell me about any experiences you've had with carrying out text analysis research with collaborators as part of a team?

- Have you approached the library, digital humanities center, or other institutional resource outside of your department/college unit for assistance with your text analysis research?

- What is your view of a collaborative/team process for textual analytics research? Research Project

4. Please walk me through a recent research project that used text analytical methods from beginning to end:

Prompts

- Purpose of research/Research question(s)

- Duration of project

- Project collaborators worked with

- Desired/actual set of texts

- Size/scope of texts 
- Algorithms/methods (if you have settled on them yet)

- Format/presentation of results (Numerical results? Plots/graphs? Other kinds of visualizations?)

5. What challenges have you encountered when conducting textual analysis?

Prompts

- Scale

- Access and Copyright

- Communication/collaboration

- Validation/reproducibility

- Dissemination

- Disciplinary culture

6. What do you do with your resulting data after you complete your analysis?

Prompts

- How have your textual analytics research been received by colleagues in your disciplinary field?

- Do you perceive any barriers to disseminating your textual analytics research in the primary journals/publication outlets for your field?

- Reproducibility/reuse

\section{Teaching with Text Analysis Tools and Broader Needs}

7. How have you used text analysis in your teaching?

Prompts

- Why did you choose to use text analysis in your course(s)?

- Have you used any of the text analysis tools or services provided by the HathiTrust Research Center in your classes?

- When has it been effective to integrate text analysis into your course curriculum?

- How does your text analysis approach fit with the learning outcomes for your discipline?

8. What tools would you be most interested in using for text analysis?

Prompts

- Have you used any of the text-analytic tools or services provided the HathiTrust Research Center? If so, which ones?

- Can you suggest a few additional text analytic tools/services/algorithms/resources that you are likely to find useful in the context of HTRC?

- Do you use probabilistic algorithms?

- Do you assess statistical significance?

- Do the tools you'd be interested in working with allow you to set parameters?

- If so, what criteria do you use to select parameters?

\section{Notes}

1. Taxonomy of Digital Research Activities in the Humanities website, "Collaboration" definition, available online at https://github.com/dhtaxonomy/TaDiRAH/blob/master/reference/activities.md [accessed 29 June 2019].

2. Jennifer Vinopal and Monica McCormick. "Supporting Digital Scholarship in Research Libraries: Scalability and Sustainability," Journal of Library Administration 53, no. 1 (2013): 27-42.

3. American Council of Learned Societies. Commission on Cyberinfrastructure for the Humanities and Social Sciences, Our Cultural Commonwealth: The Report of the American Council of Learned Societies Commission on Cyberinfrastructure for the Humanities and Social Sciences (New York, NY: American Council of Learned Societies, 2006). 
4. See, for example, Jennifer Edmond, "Collaboration and Infrastructure," in A New Companion to Digital Humanities, eds. Susan Schreibman, Raymond George Siemens, and John Unsworth (Malden, MA: Wiley-Blackwell, 2016), 54-65.

5. Lynne Siemens, "'It's a Team if You Use "Reply All": An Exploration of Research Teams in Digital Humanities Environments," Literary and Linguistic Computing 24, no. 2 (2009): 225-33.

6. Lynne Siemens, "The Balance between On-line and In-person Interactions: Methods for the Development of Digital Humanities Collaboration," Digital Studies/Le Champ Numérique 2, no. 1 (2011).

7. Tanya Clement and Doug Reside, "Off the Tracks: Laying New Lines for Digital Humanities Scholars" (2011), available online at http://hdl.handle.net/1903/14731 [accessed 26 July 2019]; Bethany Nowviskie, "Where Credit Is Due: Preconditions for the Evaluation of Collaborative Digital Scholarship," Profession, no. 1 (2011): 169-81; Bethany Nowviskie, "Evaluating Collaborative Digital Scholarship (or, Where Credit Is Due)," Journal of Digital Humanities 1, no. 4 (2012): 16-30.

8. Collaborative Research in the Digital Humanities: A Volume in Honour of Harold Short, on the Occasion of His 65th Birthday and His Retirement, September 2010, eds. Marilyn Deegan and Willard McCarty (Farnham, UK: Ashgate, 2012).

9. Michael Simeone et al., “Digging into Data Using New Collaborative Infrastructures Supporting Humanities-based Computer Science Research," First Monday 16, no. 5 (2011).

10. See, for example, Lynne Siemens and INKE Research Group, "Responding to Change and Transition in INKE's Year 3," Scholarly and Research Communication 4, no. 3 (2013).

11. Quinn Dombrowski, "Whatever Happened to Project Bamboo?" Literary and Linguistic Computing 29, no. 3 (2014): 326-39.

12. Micah Vandegrift and Stewart Varner, “Evolving in Common: Creating Mutually Supportive Relationships between Libraries and the Digital Humanities," Journal of Library Administration 53, no. 1 (2013): 67-78; Alix Keener, "The Arrival Fallacy: Collaborative Research Relationships in the Digital Humanities," DHQ: Digital Humanities Quarterly 9, no. 2 (2015).

13. Vinopal and McCormick, "Supporting Digital Scholarship in Research Libraries."

14. Elías Tzoc, "Libraries and Faculty Collaboration: Four Digital Scholarship Examples," Journal of Web Librarianship 10, no. 2 (2016): 124-36.

15. Brandon Locke and Kristen Mapes, "Do DH Librarians Need to Be in the Library? DH Librarianship in Academic Units," dh+lib: Where the Digital Humanities and Librarianship Meet (2016); Hillary A.H. Richardson and Nickoal Eichmann-Kalwara, "Process and Collaboration: Assessing Digital Humanities Work through an Embedded Lens," College \& Undergraduate Libraries 24, no. 2/4 (2017): 595-615.

16. Trevor Muñoz, “Digital Humanities in the Library Isn't a Service," Trevor Muñoz: Writing 19 (2012), available online at http://trevormunoz.com/notebook/2012/08/19/doing-dh-in-the-library.html [accessed 26 July 2019]; Bethany Nowviskie, "Skunks in the Library: A Path to Production for Scholarly R\&D," Journal of Library Administration 53, no. 1 (2013): 53-66.

17. Nisa Bakkalbasi, Damon Jaggars, and Barbara Rockenbach, "Re-skilling for the Digital Humanities: Measuring Skills, Engagement, and Learning," Library Management 36, no. 3 (2015): 208-14.

18. See: Humanities Without Walls website, available online at www.humanitieswithoutwalls.illinois.edu/ [accessed 26 July 2019] and Central New York Humanities Corridor website, available online at www.cnycorridor.net/ [accessed 26 July 2019].

19. See: National Endowment for the Humanities website, available online at https://www.neh.gov/grants/ research/collaborative-research-grants [accessed 26 July 2019] and Digging into Data Challenge website, available online at https://diggingintodata.org/ [accessed 26 July 2019].

20. Gregg Lambert, "Collaboration," in The Subject of Rosi Braidotti: Politics and Concepts, eds. Bolette Blaagaard and Iris van der Tuin (London, UK: Bloomsbury Academic, 2014), 220.

21. Julie Ellison, "This American Life: How Are the Humanities Public?" American Academy of Arts and Sciences Humanities Indicators Project 200, no. 5 (2009): 10.

22. Stephanie LeMenager and Stephanie Foote, "The Sustainable Humanities," PMLA 127, no. 3 (2012): 572-78; Regina Bendix, Kilian Bizer, and Dorothy Noyes, Sustaining Interdisciplinary Collaboration: A Guide for the Academy (Champaign, IL: University of Illinois Press, 2017).

23. Paul W. Mattessich and Kirsten M. Johnson, Collaboration: What Makes It Work, 3rd ed. (Nashville, TN: Fieldstone Alliance, 2018); William C. Barley and Nikki R. Weickum, "The Work Gap: A Structured Review of Collaborative Teamwork Research from 2005 to 2015," Annals of the International Communication Association 41, no. 2 (2017): 136-67; National Research Council, Enhancing the Effectiveness of Team Science (Washington, DC: National Academies Press, 2015). 
24. Jerry A. Jacobs and Scott Frickel, "Interdisciplinarity: A Critical Assessment," Annual Review of Sociology 35 (2009): 43-65.

25. Brian Uzzi, Satyam Mukherjee, Michael Stringer, and Ben Jones, "Atypical Combinations and Scientific Impact," Science 342, no. 6157 (2013): 468-72.

26. Juliet M. Corbin and Anselm L. Strauss, Basics of Qualitative Research: Techniques and Procedures for Developing Grounded Theory, 3rd ed. (Los Angeles, CA: Sage Publications, 2008).

27. Vinopal and McCormick, "Supporting Digital Scholarship in Research Libraries."

28. See, for example, Bridget Whearty, "Invisible in 'The Archive': Librarians, Archivists, and The Caswell Test," presented at the 53rd International Congress of Medieval Studies, Kalamazoo, MI, May 2018. 